\title{
On a roll for new TRF targets
}

\author{
Jaime H. Reina and Nouria Hernandez ${ }^{1}$ \\ Center for Integrative Genomics (CIG), Génopode Building, Faculty of Biology and Medicine, University of Lausanne, \\ 1015 Lausanne, Switzerland
}

In the early 1990s, one of us wrote in these pages a review entitled "TBP, a universal transcription factor?" (Hernandez 1993). At the time, it had become clear that the TATA-box-binding protein TBP was not a transcription factor exclusively involved in transcription from RNA polymerase II (pol II) promoters as had been thought before, but rather a factor involved in transcription by all three main types of eukaryotic nuclear RNA polymerases. In retrospect, however, the question mark at the end of the title was a wise touch! Indeed, shortly thereafter, the first TBP-related factor, TRF1, was described (Crowley et al. 1993). Since then, two more TRFs have been discovered (for review, see Berk 2000; Davidson 2003; Hochheimer and Tiian 2003), and it was found that some genes dispense with TBP and TRFs altogether (Wieczorek et al. 1998). This "expansion" of TBP into a TBP family of proteins begs the question of which promoters are targeted by which TBP family member. In this issue of Genes \& Development, Isogai et al. (2007a) report that the TATA-less histone $H 1$ promoter is regulated by TRF2. This provides a possible mechanism for earlier observations linking TRF2 with chromatin structure (Martianov et al. 2002; Kopytova et al. 2006). Furthermore, the identification by Isogai et al. (2007a) of a large number of TRF2-bound sites in the Drosophila genome helps to establish Drosophila TRF2 as a broadly used core-promoter factor.

Among the three classes of TBP-related factors described so far, TRF2 - also called TBP-like protein (TLP) or TBP-like factor (TLF) - is the only one to be widely present in metazoans (Ohbayashi et al. 1999; Kaltenbach et al. 2000; Veenstra et al. 2000). TRF1 has been found only in Drosophila and Anopheles (Crowley et al. 1993; Isogai et al. 2007b), and TRF3 is restricted to vertebrates (Persengiev et al. 2003). All three proteins contain a core domain related to the TBP C-terminal core domain, and some also contain variable $\mathrm{N}$ - and $\mathrm{C}$-terminal domains.

\section{TRF1}

TRF1 was discovered in a genetic screen for flies with a shaker phenotype, which is generally attributed to nervous system defects. In situ staining showed TRF1 to be ubiquitously expressed but differentially up-regulated in

${ }^{1}$ Corresponding author.

E-MAIL nouria.hernandez@unil.ch; FAX 41-21-692-3925.

Article is online at http://www.genesdev.org/cgi/doi/10.1101/gad.1623207. the CNS and gonads during the early stages of embryonal development (Hansen et al. 1997). TRF1 shares 63\% identity with TBP within the repeats in the C-terminal core domain (Rabenstein et al. 1999; Persengiev et al. 2003). In vitro, TRF1 can bind to TATA boxes, associate with both TFIIA and TFIIB, and efficiently replace TBP in transcription from a TATA-box-containing promoter (Hansen et al. 1997). Moreover, it can direct transcription from an alternate upstream promoter in the tudor gene, binding - together with TFIIA and TFIIB - to a TCrich sequence (Fig. 1A).

Although TRF1 was first described in association with several polypeptides in a $>500-\mathrm{kDa}$ multiprotein complex (Hansen et al. 1997), later work showed that the majority of TRF1 $(>90 \%)$ is in fact associated with the Drosophila homolog of the TFIIB-related pol III transcription factor, Brf1 (Dm-Brf1), in a $300-k D a$ complex (Takada et al. 2000). Indeed, immunodepletions of either TBP or TRF1 from a Drosophila Schneider line-2 (S2) cell transcription extract combined with in vitro transcription assays showed that TRF1, but not TBP, directs transcription by pol III. Genome-wide chromatin immunoprecipitation (ChIP) experiments confirmed that TRF1 is mostly a pol III transcription factor. Thus, these experiments identified some TRF1 targets as pol II promoters devoid of Brf1, supporting the idea that TRF1 is involved in transcription of a small subset of pol II genes whose defective transcription was perhaps responsible for the original shaker phenotype. However, most TRF1 targets were pol III promoters that also bound Brf1 (see Fig. 1; Isogai et al. 2007b). In Drosophila cells, then, the TFIIIB activity, which is the key factor responsible for pol III recruitment (for a review, see Schramm and Hernandez 2002), contains Brf1 and TRF1, whereas in yeast it contains Brf1 and TBP. Moreover, human cells contain two TFIIIB activities involved in transcription of distinct classes of pol III genes, one corresponding to yeast TFIIIB containing human Brfl and TBP, and a second one in which Brf1 is replaced by another TFIIB-related factor called Brf2 (Teichmann et al. 1999; Schramm et al. 2000). It seems likely that there are many more, so far unsuspected variations of the basal pol III transcription machinery.

\section{TRF3}

TRF3, the most recently discovered TRF, has been characterized in human (Persengiev et al. 2003), mouse 
Figure 1. Targets of TBP and TRFs in Drosophila and vertebrates. (A) Schematic depiction of various promoters in Drosophila. (TBP panel) A pol II promoter containing a TATA-box (yellow) with bound TFIID upstream of the transcription start site (arrow). (TRF1 panel, top model) A pol III promoter with the TRF1Brf1 complex bound to a region (green) upstream of the start site (binding to this region is mediated by accessory factors such as TFIIIA and/or TFIIIC, not shown here). (TRF1 panel, bottom model) The tudor gene tandem promoters with a TRF1-TAFs complex bound to a TC-rich element (blue) to direct transcription from an upstream start site, and TFIID bound to a TATA-box to direct transcription from the downstream start site. (TRF2 panel) The tandem PCNA promoters with DREF associated with TRF2 and other polypeptides-in particular, some subunits also present in the NURF complex, bound to a $D R E$ (purple) to direct transcription from an upstream start site, and TFIID bound to the TATA-less downstream promoter to direct transcription from a downstream site. $(B)$ Schematic depiction of various promoters in vertebrates. (TBP panel) A pol II promoter containing a TATA-box (yellow) with bound TFIID upstream of the transcription start site. (TRF3 panel, top model) A promoter from a gene involved in embryogenesis with the TRF3-TAFs complex bound to a TATA-box (yellow). (TRF3 panel, bottom model) A promoter from a gene such as myogenin required for muscle differentiation bound by a TRF3-TAF3 complex, which replaces TFIID during myogenesis. (TRF2 panel, top model) The TATA-less NF-1 promoter with a TRF2-TFIIA complex containing perhaps additional polypeptides bound to an undefined promoter element (blue). (TRF2 panel, bottom model) A promoter from a gene involved in gametogenesis with a TRF2-TFIIA/ ALF complex containing perhaps additional polpeptides bound to an undefined promoter element (blue). (C) Newly identified Drosophila TRF2 targets. The top panel shows the Drosophila histone gene cluster: the core-histone genes $H 2 A, H 2 B, H 3$, and $H 4$ with TFIID bound to the TATA-box (yellow), and the linker histone H1 TATA-less gene with a TRF2 complex containing TFIIA and perhaps other polypeptides bound to a TCrich region (blue). The bottom panel shows riboprotein gene promoters with TRF2 and hypothetical TAFs bound to an undefined promoter element (blue).

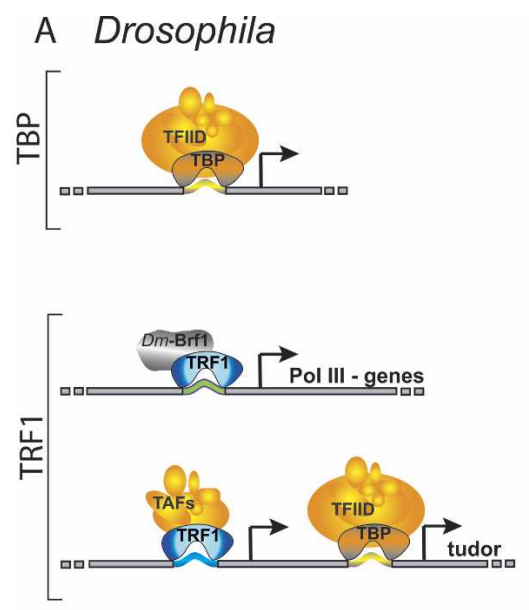

B Vertebrates
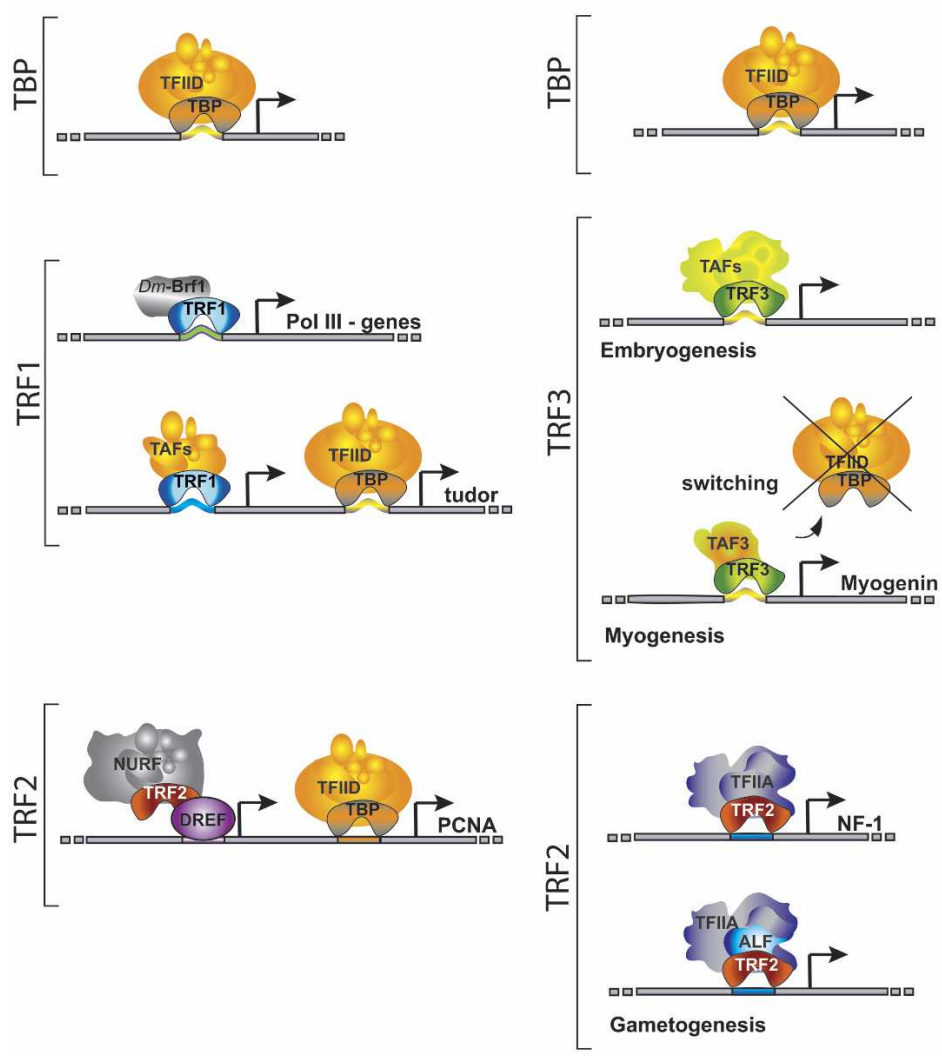

C Drosophila
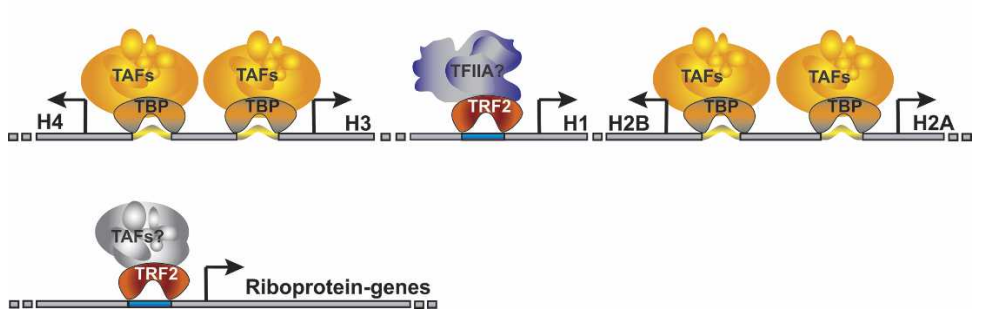

(Deato and Tjian 2007), and Xenopus (Jallow et al. 2004) cells, and corresponding expressed sequence tags (ESTs) have also been identified in fish and other vertebrates. It is the TRF most closely related to TBP and it is broadly expressed in different cell types (Persengiev et al. 2003). Like TBP and TRF1, TRF3 is able to bind to TATA boxes together with TFIIA and TFIIB, and it can promote transcription in vitro from TATA-box-containing promoters (Bartfai et al. 2004; Jallow et al. 2004). It is essential for embryogenesis in Xenopus and zebrafish (Bartfai et al. 2004; Jallow et al. 2004). In a recent study using the mouse myoblast cell line $\mathrm{C} 2 \mathrm{C} 12$ (which can be differentiated into myotubes), as well as primary myoblasts from newborn mice and myofibers from adult mice, TRF3 was shown to form a 150 - to $200-\mathrm{kDa}$ complex with TBP- associated factor 3 (TAF3). Remarkably, this complex replaces the canonical TFIID complex in myotubes, which appear nearly devoid of TFIID (see Fig. 1B), and is required for differentation of $\mathrm{C} 2 \mathrm{C} 12$ myoblasts into myotubes (Deato and Tiian 2007). Moreover, the TRF3-TAF3 complex is enriched on the Myogenin promoter in $\mathrm{C} 2 \mathrm{C} 12$ myotubes as compared with $\mathrm{C} 2 \mathrm{C} 12$ myoblasts, and appears on this promoter within $24 \mathrm{~h}$ of differentiation induction. Thus, differentiation of muscle cells is accompanied by a general depletion of TFIID and replacement, at least at some key promoters, by TRF3TAF3. This raises the possibility that the replacement of TFIID with TRF3-TAF3 is a more general feature of differentiating cells, and that TBP-containing complexes involved in pol I and pol III transcription in actively di- 
viding cells may also be replaced by other, perhaps TRF3containing, complexes (Deato and Tjian 2007).

\section{TRF2}

TRF2 was discovered by analysis of ESTs by several groups and, accordingly, variously named TRF2 (Rabenstein et al. 1999; Teichmann et al. 1999), TRF (Maldonado 1999), TLP (Ohbayashi et al. 1999, 2001), TLF (Kaltenbach et al. 2000; Veenstra et al. 2000), or TBPrelated protein (TRP) (Moore et al. 1999). Sequence alignments and phylogenetic analyses indicate that the TRF2s from a number of metazoans group together in a family that is more distantly related to TBP than TRF1 (Dantonel et al. 1999). As a family, the TRF2s are less conserved than the TBPs: The TRF2 core domains are $\sim 40 \%$ identical to TBP core domains, but TRF2 core domains are $40 \%-45 \%$ identical among metazoans, whereas TBP core domains are $80 \%$ identical from yeast to human (Dantonel et al. 2000). Although the high structural similarity $(-60 \%)$ between TRF2 and TBP core domains suggests that they adopt the same saddle-like structure, the TBP residues required for TATA-box binding are not conserved in TRF2, suggesting that TRF2 requires other elements to bind DNA (see Berk 2000 and references therein). Indeed, TRF2 does not detectably bind to TATA boxes in vitro (Moore et al. 1999; Rabenstein et al. 1999; Teichmann et al. 1999). It does, however, associate with TFIIA and TFIIB (Moore et al. 1999), and in fact is found complexed with TFIIA in a HeLa cell line expressing a tagged TRF2, and with TFIIA-like factor (ALF), a TFIIA paralog expressed in male germ cells (Teichmann et al. 1999; Catena et al. 2005; Kopytova et al. 2006). TRF2 is unable to replace TBP or TFIID function in basal or activated pol II transcription in vitro. Rather, it can inhibit TBP and TFIID-mediated transcription in such assays, probably in part by sequestering TFIIA (Moore et al. 1999; Teichmann et al. 1999; Nakadai et al. 2004). Moreover, it cannot replace TBP for pol III transcription in extracts from human cells, at least for those genes that recruit the Brf1-containing TFIIIB activity (Teichmann et al. 1999).

\section{Which, then, are the targets of TRF2?}

The first TRF2 targets were identified as a result of coimmunoprecipitation studies, in which Drosophila TRF2 was found to associate with as many as 18 polypeptides, among them a few components of the NURF remodeling complex, including the catalytic ATPase subunit ISWI and the DNA replication-related elementbinding factor (DREF) (Hochheimer et al. 2002). DREF was known to be involved in the regulation of cell cycle and cell proliferation genes such as the proliferating cell nuclear antigen (PCNA) gene by binding to the DRE (Hirose et al. 1993). In vitro and in cell assays then established that the PCNA gene contains two different transcription start sites: a downstream start site controlled by the TFIID complex through a TATA-less element, and an upstream start site controlled by the TRF2/DREF complex through a DRE (see Fig. 1; Hochheimer et al.
2002). Interestingly, a computational analysis identified the DRE as one of the prevalent core-promoter motifs in the Drosophila genome, raising the possibility that the TRF2/DREF-containing complex controls a large group of promoters (Ohler et al. 2002; Tomancak et al. 2002). As described below, this is indeed most probably the case.

In human cells, overexpression of TRF2 was found to activate transcription of the TATA-less neurofibromatosis type 1 gene (NF-1). This effect was dependent on its ability to bind TFIIA as determined by overexpression of a mutant TRF2 unable to bind to TFIIA. Reciprocally, overexpression of TRF2 repressed transcription from the TATA-box-containing c-fos gene, probably by sequestering TFIIA (Chong et al. 2005). Similarly, chicken TRF2 was shown to inhibit transcription from TATA-box-containing promoters while stimulating transcription from TATA-less promoters, and mouse TRF2 to inhibit transcription of the wee1 gene, in both cases again in a manner dependent on TRF2's ability to bind to TFIIA (Ohbayashi et al. 2003; Tanaka et al. 2007).

\section{TRF2 targets the histone $\mathrm{H} 1$ gene within the histone gene cluster}

In invertebrates, the genes encoding the replicationdependent core histone proteins $\mathrm{H} 2 \mathrm{~A}, \mathrm{H} 2 \mathrm{~B}, \mathrm{H} 3$, and $\mathrm{H} 4$, as well as the linker histone $\mathrm{H} 1$ are organized in tandem repeats that can number up to $\sim 100$ copies per haploid genome. In higher eukaryotes the organization is different in that the histone genes are more dispersed even though some clusters occur, and the number of copies per haploid genome is lower (for review, see Osley 1991). These five histones genes are transcribed by pol II, but they lack introns and polyadenylation sites, such that the pre-mRNAs are neither spliced nor polyadenylated. Rather, they terminate with a well-conserved stem-andloop structure that regulates their maturation, translation, and stability (Marzluff 2005).

In this issue, Isogai, et al. (2007a) report the identification of histone $H 1$ as a target of TRF2, suggesting that histone genes within a cluster can be differentially regulated by selective usage of different core transcriptional machineries. In polytene chromosome stainings with antibodies against TBP or TRF2, they noticed that the two proteins displayed a largely distinct pattern of occupancy except for a locus proximal to the chromocenter, where TBP and TRF2 colocalized. The localization of TRF2 to this locus appeared DREF independent, as no DREF could be detected. The cytological location suggested that the locus might correspond to the histone gene cluster, which in Drosophila contains about a hundred copies of the histone genes, a hypothesis that could be confirmed by fluorescent in situ hybridization (FISH). Indeed, in cultured interphase cells, the histone gene cluster is known to localize to a structure called the histone locus body (HLB) (Liu et al. 2006). Immunostaining of Drosophila S2 and Kc cells confirmed that TBP and TRF2 colocalize to a structure also stained by an anti-2,2,7trimethyl guanosine antibody, an antibody recognizing the cap structure of small nuclear RNAs marking the 
HLB (Liu et al. 2006). ChIPs with synchronized S-phase cells then showed selective binding of TBP and TAF2, a TFIID subunit, to the core histone gene promoters and TRF2 to the H1 promoter. In contrast, pol II and TFIIA were present on all five promoters. Pol II and, to a lesser extent, TBP were preferentially bound to core histone promoters during $S$ phase, whereas TRF2 was bound to the $H 1$ promoter during all phases of the cell cycle. Unfortunately, the TFIIA status during the cell cycle was not determined.

The functional significance of TRF2 localization on the $H 1$ promoter was addressed by RNA interference (RNAi) and rescue experiments in S2 cells. These experiments showed that depletion of TRF2, but not TBP or the TFIID subunit TAF5, reduced both endogenous histone $\mathrm{H} 1 \mathrm{mRNA}$ levels and the activity of a histone $H 1$ promoter reporter construct. Moreover, the activity could be restored by inducible expression of a tagged TRF2 protein. Depletion of DREF had a much more modest effect, which, given the absence of DREs in the histone cluster, are attributed to indirect effects. Mapping of cis-elements required for TRF2-dependent histone $H 1$ transcription identified a critical TC-rich element between -55 and -40 , although the element does not appear to be recognized by TRF2 alone. Thus, the TATAless histone $H 1$ promoter is an example of a promoter uniquely regulated by TRF2 and not by TBP.

Genome-wide screening for sites occupied by TRF2, TBP, and pol II in S2 cells was then undertaken. ChIPon-chip experiments revealed more than a thousand sites occupied by TRF2, $60 \%$ of which were also occupied by pol II. TBP and TRF2 sites were interspersed in most of the genome except for chromosome 4, which showed only four TRF2 sites and 95 TBP sites, most of them, curiously, devoid of pol II. Although the majority of TRF2-occupied sites were nonoverlapping with TBP-occupied sites, $20 \%$ did overlap, suggesting that tandem promoters of the PCNA type are quite frequent in the Drosophila genome. Interestingly, among the TRF2 targets is a cluster of ribosomal protein genes that are probably coregulated. Analysis of the TRF2-occupied core promoters for the presence of sequence motifs revealed that TRF2 targets are mostly devoid of TATA boxes and enriched for DREs, the binding site for DREF (see above), but no other striking motif localized at a fixed distance from the transcription start site was identified. Altogether, this analysis reveals that a large number of genes in the Drosophila genome are probably controlled by TRF2 rather than TBP.

Consistent with a broad role of TRF2, TRF2-deficient flies are not viable, and partial TRF2 inactivation leads to various defects such as impaired premeiotic chromatin condensation and differentiation of male and female germ cells (Kopytova et al. 2006; Shima et al. 2007). To gain further insight into the role of TRF2 in vivo, Isogai et al. (2007a) used RNAi to decrease TRF2 levels specifically in salivary glands. The embryos developed normally up to the third instar larval stage (L3), but then failed to pupate or died during pupal stages.

TRF2-depleted salivary glands of L3 embryos were much smaller than normal, an effect due to reduced cell size rather than reduced cell number. The chromatin structure of polytene chromosomes was severely disrupted, with a lack of clear banding pattern and numerous regions of thin chromatin fibers suggestive of improperly condensed chromatin, and this was correlated with much reduced levels of both histone $\mathrm{H} 1 \mathrm{mRNA}$ and protein. Moreover, the expression of a dozen ribosomal protein genes identified as TRF2 targets in the genomewide survey was much diminished, which may relate to the defect in cell size. Curiously, however, expression from the PCNA promoter was unaffected, suggesting that the TBP-dependent downstream PCNA core promoter is sufficient for efficient PCNA expression in the absence of TRF2.

The identification of the histone $\mathrm{H} 1$ genes as genes regulated by TRF2 is remarkable, given that these genes are located within a cluster of core histone genes, all regulated by TBP. This selective use of different basalpromoter factors probably confers to the cell a mechanism by which to alter the ratio of core and linker histones and thus regulate some aspects of chromatin structure. Indeed, the observation that TRF2 depletion leads to both reduced amounts of histone $H 1$ and altered polytene chromosomes provides further evidence that varying levels of histone $H 1$ can have dramatic effects on chromatin structure (Fan et al. 2005).

The identification of more than a thousand sites bound by TRF2 in the Drosophila genome establishes Drosophila TRF2 as a major regulator of TATA-less promoters. This is consistent with studies in nematode, fly, fish, and frog, where inactivation of TRF2 results in a block in embryonic development and lethality (Dantonel et al. 2000; Kaltenbach et al. 2000; Veenstra et al. 2000; Bartfai et al. 2004; Kopytova et al. 2006). However, inactivation of TRF2 in the mouse has no major detrimental effects except for male sterility, resulting from impaired spermiogenesis (Zhang et al. 2001). Extensive cytological analysis of these null mice revealed a defect in chromatin condensation in early spermatids, suggesting a function of TRF2 in chromatin organization, in particular in the formation of chromocenter structures (Martianov et al. 2002). Is TRF2 then only involved in the expression of a small group of genes in mouse and other mammalian cells? This is possible; indeed, the precedent of TBP, which in insect cells does not participate in pol III transcription, illustrates how members of the TBP family can perform different functions in different species. Another possibility is that TRF2 does in fact regulate transcription of a large number of genes in mammalian cells, but that its function can be taken over by TBP when it is missing.

In the last 15 years, we have come to realize that the pol II basal transcription machinery is much more varied than was originally thought. It is likely that such variation will extend to the pol I and pol III basal machineries, which have been, so far, studied mostly in cell-free extracts and cultured cells. Indeed, it seems that nature does not neglect any mechanism that can lead to added levels of regulation! 


\section{Acknowledgments}

This work was funded by the University of Lausanne and by SNSF grant 3100A0-109941/1.

\section{References}

Bartfai, R., Balduf, C., Hilton, T., Rathmann, Y., Hadzhiev, Y., Tora, L., Orban, L., and Muller, F. 2004. TBP2, a vertebratespecific member of the TBP family, is required in embryonic development of zebrafish. Curr. Biol. 14: 593-598.

Berk, A.J. 2000. TBP-like factors come into focus. Cell 103: 5-8. Catena, R., Argentini, M., Martianov, I., Parello, C., Brancorsini, S., Parvinen, M., Sassone-Corsi, P., and Davidson, I. 2005. Proteolytic cleavage of ALF into $\alpha$ - and $\beta$-subunits that form homologous and heterologous complexes with somatic TFIIA and TRF2 in male germ cells. FEBS Lett. 579: 34013410.

Chong, J.A., Moran, M.M., Teichmann, M., Kaczmarek, J.S., Roeder, R., and Clapham, D.E. 2005. TATA-binding protein (TBP)-like factor (TLF) is a functional regulator of transcription: Reciprocal regulation of the neurofibromatosis type 1 and c-fos genes by TLF/TRF2 and TBP. Mol. Cell. Biol. 25: 2632-2643.

Crowley, T.E., Hoey, T., Liu, J.K., Jan, Y.N., Jan, L.Y., and Tiian, R. 1993. A new factor related to TATA-binding protein has highly restricted expression patterns in Drosophila. Nature 361: 557-561.

Dantonel, J.C., Wurtz, J.M., Poch, O., Moras, D., and Tora, L. 1999. The TBP-like factor: An alternative transcription factor in metazoa? Trends Biochem. Sci. 24: 335-339.

Dantonel, J.C., Quintin, S., Lakatos, L., Labouesse, M., and Tora, L. 2000. TBP-like factor is required for embryonic RNA polymerase II transcription in C. elegans. Mol. Cell 6: 715722.

Davidson, I. 2003. The genetics of TBP and TBP-related factors. Trends Biochem. Sci. 28: 391-398.

Deato, M.D. and Tjian, R. 2007. Switching of the core transcription machinery during myogenesis. Genes \& Dev. 21: 21372149.

Fan, Y., Nikitina, T., Zhao, J., Fleury, T.J., Bhattacharyya, R., Bouhassira, E.E., Stein, A., Woodcock, C.L., and Skoultchi, A.I. 2005. Histone H1 depletion in mammals alters global chromatin structure but causes specific changes in gene regulation. Cell 123: 1199-1212.

Hansen, S.K., Takada, S., Jacobson, R.H., Lis, J.T., and Tjian, R. 1997. Transcription properties of a cell type-specific TATAbinding protein, TRF. Cell 91: 71-83.

Hernandez, N. 1993. TBP, a universal eukaryotic transcription factor? Genes \& Dev. 7: 1291-1308.

Hirose, F., Yamaguchi, M., Handa, H., Inomata, Y., and Matsukage, A. 1993. Novel 8-base pair sequence (Drosophila DNA replication-related element) and specific binding factor involved in the expression of Drosophila genes for DNA polymerase $\alpha$ and proliferating cell nuclear antigen. J. Biol. Chem. 268: 2092-2099.

Hochheimer, A. and Tjian, R. 2003. Diversified transcription initiation complexes expand promoter selectivity and tissuespecific gene expression. Genes \& Dev. 17: 1309-1320.

Hochheimer, A., Zhou, S., Zheng, S., Holmes, M.C., and Tjian, R. 2002. TRF2 associates with DREF and directs promoterselective gene expression in Drosophila. Nature 420: 439445.

Isogai, Y., Keles, S., Prestel, M., Hochheimer, A., and Tjian, R. 2007a. Transcription of histone gene cluster by differential core-promoter factors. Genes \& Dev. (this issue), doi:
$10.1101 /$ gad.1608807.

Isogai, Y., Takada, S., Tjian, R., and Keles, S. 2007b. Novel TRF1/BRF target genes revealed by genome-wide analysis of Drosophila Pol III transcription. EMBO J. 26: 79-89.

Jallow, Z., Jacobi, U.G., Weeks, D.L., Dawid, I.B., and Veenstra, G.J. 2004. Specialized and redundant roles of TBP and a vertebrate-specific TBP paralog in embryonic gene regulation in Xenopus. Proc. Nat1. Acad. Sci. 101: 13525-13530.

Kaltenbach, L., Horner, M.A., Rothman, J.H., and Mango, S.E. 2000. The TBP-like factor CeTLF is required to activate RNA polymerase II transcription during C. elegans embryogenesis. Mol. Cell 6: 705-713.

Kopytova, D.V., Krasnov, A.N., Kopantceva, M.R., Nabirochkina, E.N., Nikolenko, J.V., Maksimenko, O., Kurshakova, M.M., Lebedeva, L.A., Yerokhin, M.M., Simonova, O.B., et al. 2006. Two isoforms of Drosophila TRF2 are involved in embryonic development, premeiotic chromatin condensation, and proper differentiation of germ cells of both sexes. Mol. Cell. Biol. 26: 7492-7505.

Liu, J.L., Murphy, C., Buszczak, M., Clatterbuck, S., Goodman, R., and Gall, J.G. 2006. The Drosophila melanogaster Cajal body. J. Cell Biol. 172: 875-884.

Maldonado, E. 1999. Transcriptional functions of a new mammalian TATA-binding protein-related factor. J. Biol. Chem. 274: 12963-12966.

Martianov, I., Brancorsini, S., Gansmuller, A., Parvinen, M., Davidson, I., and Sassone-Corsi, P. 2002. Distinct functions of TBP and TLF/TRF2 during spermatogenesis: Requirement of TLF for heterochromatic chromocenter formation in haploid round spermatids. Development 129: 945-955.

Marzluff, W.F. 2005. Metazoan replication-dependent histone mRNAs: A distinct set of RNA polymerase II transcripts. Curr. Opin. Cell Biol. 17: 274-280.

Moore, P.A., Ozer, J., Salunek, M., Jan, G., Zerby, D., Campbell, S., and Lieberman, P.M. 1999. A human TATA binding protein-related protein with altered DNA binding specificity inhibits transcription from multiple promoters and activators. Mol. Cell. Biol. 19: 7610-7620.

Nakadai, T., Shimada, M., Shima, D., Handa, H., and Tamura, T.A. 2004. Specific interaction with transcription factor IIA and localization of the mammalian TATA-binding proteinlike protein (TLP/TRF2/TLF). J. Biol. Chem. 279: 7447-7455.

Ohbayashi, T., Makino, Y., and Tamura, T.A. 1999. Identification of a mouse TBP-like protein (TLP) distantly related to the Drosophila TBP-related factor. Nucleic Acids Res. 27: 750-755.

Ohbayashi, T., Shimada, M., Nakadai, T., and Tamura, T.A. 2001. TBP-like protein (TLP/TLF/TRF2) artificially recruited to a promoter stimulates basal transcription in vivo. Biochem. Biophys. Res. Commun. 285: 616-622.

Ohbayashi, T., Shimada, M., Nakadai, T., Wada, T., Handa, H., and Tamura, T. 2003. Vertebrate TBP-like protein (TLP/ TRF2/TLF) stimulates TATA-less terminal deoxynucleotidyl transferase promoters in a transient reporter assay, and TFIIA-binding capacity of TLP is required for this function. Nucleic Acids Res. 31: 2127-2133.

Ohler, U., Liao, G.C., Niemann, H., and Rubin, G.M. 2002. Computational analysis of core promoters in the Drosophila genome. Genome Biol. 3: RESEARCH0087.1RESEARCH0087.12. doi: 10.1186/gb-2002-3-12-research0087.

Osley, M.A. 1991. The regulation of histone synthesis in the cell cycle. Annu. Rev. Biochem. 60: 827-861.

Persengiev, S.P., Zhu, X., Dixit, B.L., Maston, G.A., Kittler, E.L., and Green, M.R. 2003. TRF3, a TATA-box-binding proteinrelated factor, is vertebrate-specific and widely expressed. Proc. Nat1. Acad. Sci. 100: 14887-14891. 
Reina and Hernandez

Rabenstein, M.D., Zhou, S., Lis, J.T., and Tjian, R. 1999. TATA box-binding protein (TBP)-related factor 2 (TRF2), a third member of the TBP family. Proc. Natl. Acad. Sci. 96: 47914796.

Schramm, L. and Hernandez, N. 2002. Recruitment of RNA polymerase III to its target promoters. Genes \& Dev. 16: 2593-2620.

Schramm, L., Pendergrast, P.S., Sun, Y., and Hernandez, N. 2000. Different human TFIIIB activities direct RNA polymerase III transcription from TATA-containing and TATAless promoters. Genes \& Dev. 14: 2650-2663.

Shima, S., Aigaki, T., Nojima, T., and Yamamoto, D. 2007. Identification of trf2 mutants of Drosophila with defects in anterior spiracle eversion. Arch. Insect Biochem. Physiol. 64: 157-163.

Takada, S., Lis, J.T., Zhou, S., and Tjian, R. 2000. A TRF1:BRF complex directs Drosophila RNA polymerase III transcription. Cell 101: 459-469.

Tanaka, Y., Nanba, Y.A., Park, K.A., Mabuchi, T., Suenaga, Y., Shiraishi, S., Shimada, M., Nakadai, T., and Tamura, T.A. 2007. Transcriptional repression of the mouse weel gene by TBP-related factor 2. Biochem. Biophys. Res. Commun. 352: 21-28.

Teichmann, M., Wang, Z., Martinez, E., Tjernberg, A., Zhang, D., Vollmer, F., Chait, B.T., and Roeder, R.G. 1999. Human TATA-binding protein-related factor-2 (hTRF2) stably associates with hTFIIA in HeLa cells. Proc. Natl. Acad. Sci. 96: 13720-13725.

Tomancak, P., Beaton, A., Weiszmann, R., Kwan, E., Shu, S., Lewis, S.E., Richards, S., Ashburner, M., Hartenstein, V., Celniker, S.E. et al. 2002. Systematic determination of patterns of gene expression during Drosophila embryogenesis. Genome Biol. 3: RESEARCH0088.1-RESEARCH0088.14. doi: 10.1186/gb-2002-3-12-research0088.

Veenstra, G.J., Weeks, D.L., and Wolffe, A.P. 2000. Distinct roles for TBP and TBP-like factor in early embryonic gene transcription in Xenopus. Science 290: 2312-2315.

Wieczorek, E., Brand, M., Jacq, X., and Tora, L. 1998. Function of TAF(II)-containing complex without TBP in transcription by RNA polymerase II. Nature 393: 187-191.

Zhang, D., Penttila, T.L., Morris, P.L., Teichmann, M., and Roeder, R.G. 2001. Spermiogenesis deficiency in mice lacking the Trf2 gene. Science 292: 1153-1155. 


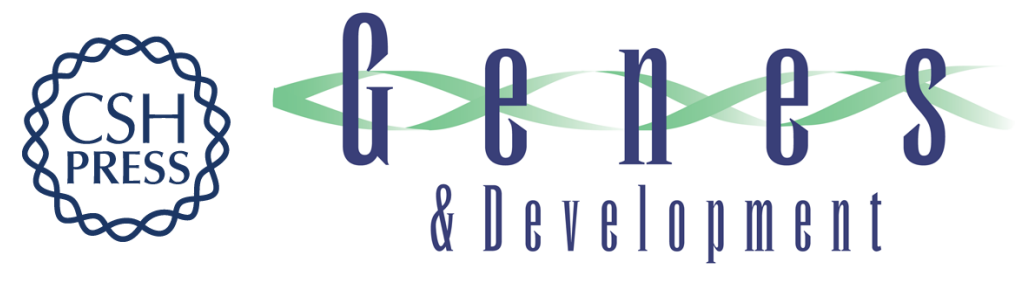

\section{On a roll for new TRF targets}

Jaime H. Reina and Nouria Hernandez

Genes Dev. 2007, 21:

Access the most recent version at doi:10.1101/gad.1623207

Related Content Transcription of histone gene cluster by differential core-promoter factors Yoh Isogai, Sündüz Keles, Matthias Prestel, et al.

Genes Dev. November , 2007 21: 2936-2949

References This article cites 43 articles, 19 of which can be accessed free at: http://genesdev.cshlp.org/content/21/22/2855.full.html\#ref-list-1

Articles cited in:

http://genesdev.cshlp.org/content/21/22/2855.full.html\#related-urls

License Freely available online through the Genes \& Development Open Access option.

Email Alerting Receive free email alerts when new articles cite this article - sign up in the box at the top Service right corner of the article or click here.

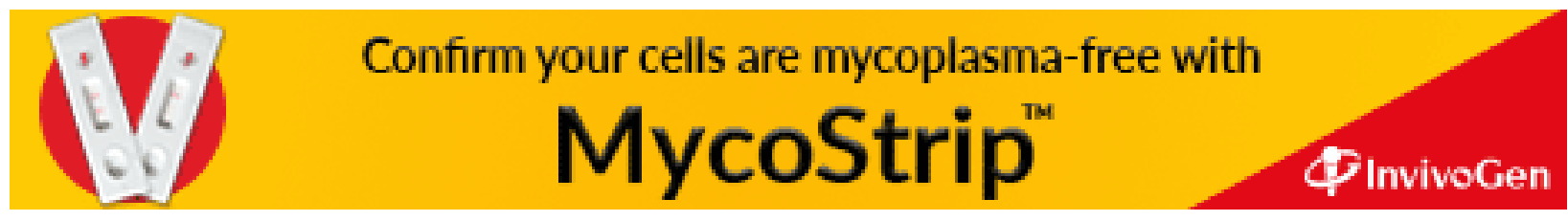

